\title{
Support for Create 3D Computer Graphics Images in GIS Systems Using Externals Renders Algorithm
}

\section{Visualization Capabilities Results of Analyzes in GIS Programs}

Browsing the possibilities posed by GIS and CAD programs in the modeling analysis results we can find many similarities and differences. Features arise from aims for which these types of programs have been developed. Goal of GIS software is to do different types of spatial analysis. A secondary aim is to do visualization of the results generated. A secondary aim is to doing visualization of the results generated. CAD software is taking data presentation proposed as an overriding goal, however, analysis tools, topology and relationship to the database are limited to special overlays to the main program or did not exist. Knowing how to do the main task of particular programs we can say that the CAD software is better suited for the visualization of data than GIS applications.

We will attempt to analyze possibility of these two types of software with regard to ArcGIS Version 10 and CAD graphics software Cinema4D R13.

For visualization in the program ArcGIS we can use two 3D environments: ArcGlobe and ArcScene. They are designed for different uses. After starting ArcGlobe application, we can see the globe, suggests to us, that all analysis and visualization will involve a large area of land. The data can be presented on a sphere Earth. ArcScene is designed for analysis and visualization covering a small area of land. Data are presented on the local reference plane. Differences also include data that we can use for analysis and visualization. In ArcGlobe, all data can be displayed in the specified simplification, depending on the magnification. This eliminates the problem of overloading the computer memory, we do not have to resign from less important data available. In ArcScene, all available data are automatically stored in the computer memory, we have quick access to them. The downside of

* AGH University of Science and Technology 
this is dependence of the maximum amount of data used to the quantities computer memory. Exceeding the limit of memory deteriorates the smooth operation of program. Many other differences between ArcGlobe and ArcScene software presented in the Table 1.

After comparing the options above we can conclude that the best tool for visualizing data on a small range, for example, the analysis of place of the buildings and the analysis of hydrographic, is a software ArcScene.

Table 1. Selected differences between ArcGlobe and ArcScene applications

\begin{tabular}{|l|c|c|}
\hline \multicolumn{1}{|c|}{ Software variants } & ArcGlobe & ArcScene \\
\hline \hline Ability to perform 3D analysis, extension 3D Analyst & Yes & No \\
\hline The ability to support large amounts of data by caching & Yes & No \\
\hline Ability to present data on the surface of the globe & Yes & No \\
\hline $\begin{array}{l}\text { Dynamic shading of depends on the definition of the position of the main } \\
\text { source of lighting }\end{array}$ & No & Yes \\
\hline Chance of presentation images as anaglyph, stereoscopic images & No & Yes \\
\hline Loading spatial data with no defined reference system, the local system & No & Yes \\
\hline Possibility of programming images animation & No & Yes \\
\hline
\end{tabular}

In the settings display quality graphics in ArcGlobe and ArcScene is available only poorly developed illumination option responsible for the lighting. It includes elements such as the angle of incidence of solar radiation, altitude and position of the simulated sun, and contrast. Poor number of options theory gives us to understand that the generated images are deviate significantly from the required photo realism.

ArcScene software will be analyzed as a dedicated tool for visualizing data on a small range for example, the analysis of the location the building and the analysis of hydrographic. In ArcScene, layers can be textured by any images using the symbolization. Texture on one layer may differ between themselves after a proper classification, or use of unique symbols for the layer arising from the database. Lighting the whole scene consists in dimming or brightening the textures depending on the angle and height of the sun. The big downside is the lack of shadows cast by the illuminated object on the other layers, such as for example, houses do not cast shadows on the ground, what makes the visualized scene the artificial. An additional problem is the lack of antialising settings, i.e. smoothing the edges of objects. This minus may be removed by setting the appropriate option in the video card drivers. This will cause partial smoothing out all the edges of the escalator. Another downsides of the ArcScene are limited opportunities of modeling objects. A simple 3D model can be created from 2D polygon by extrude. Extrusion turns polygons into blocks. 
Inserting complex shapes through the symbols is associated with the use of CAD program or the choice of a standard database of objects, such as for example: buildings, trees, cars, skyscrapers, special buildings, other structural elements of streets.

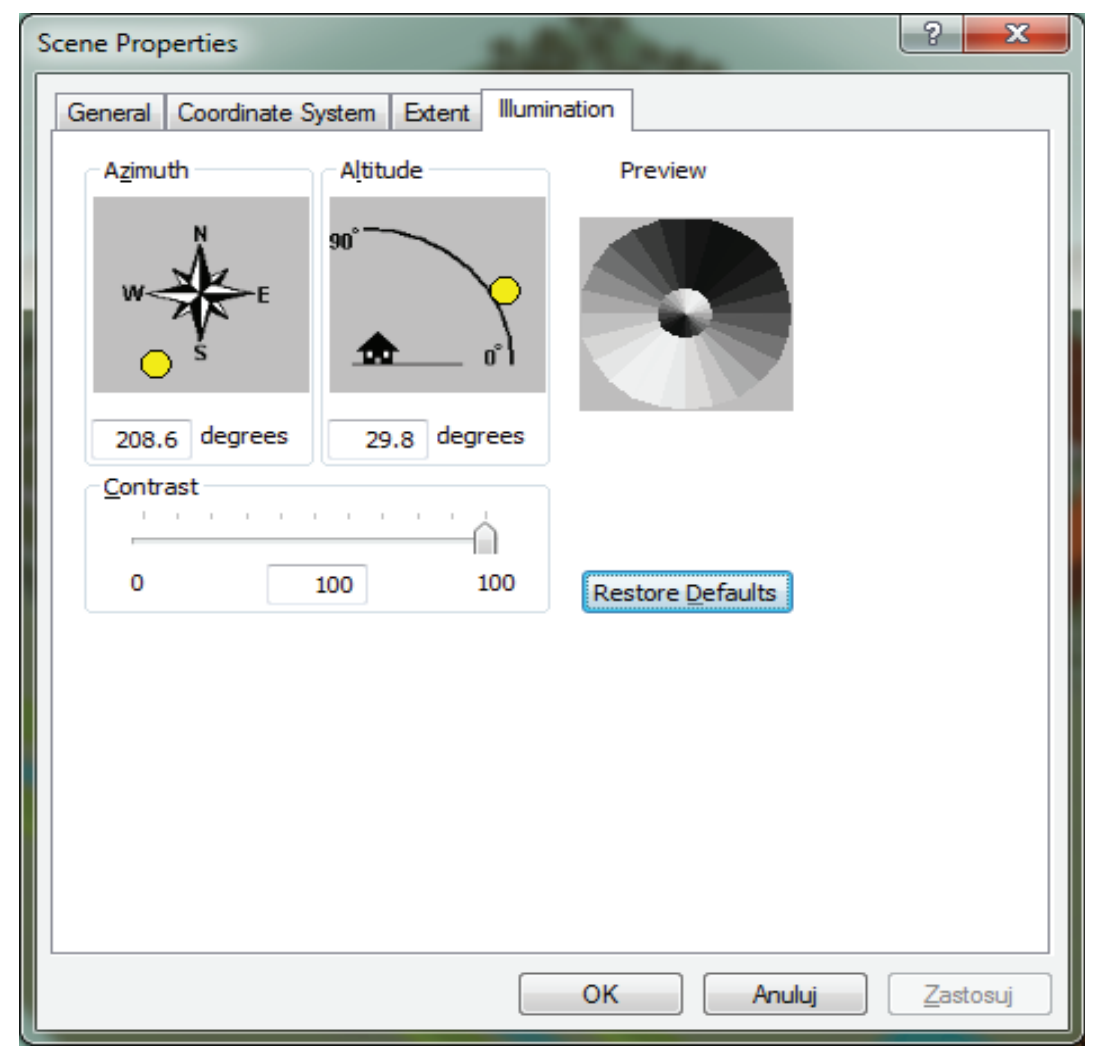

Fig. 1. Options scene illumination in the program ESRI ArcScene

In summary, the visualization software ArcScene based only on choosing the right color layers, textures, applied any symbols or models for point objects or line, determining the transparency of some layers, and the modeling of lighting scenes based on approximate setting of the sun. Setting the lighting we have to choose from three options (Fig. 1): position of the light source, the angle of incidence of the sun's rays and contrast levels. Generate simple graphics have its advantages: for larger areas of visualization does not need powerful computers in order to images were created in real time. Any change colors, textures and symbols can all the time keep track on the screen. We can add your own redefined symbols and 3D objects. In Figure 2, we can see a sample view of the planned investment, its location and plan of individual objects in 3D [3] designed in ArcScene 10 software. 


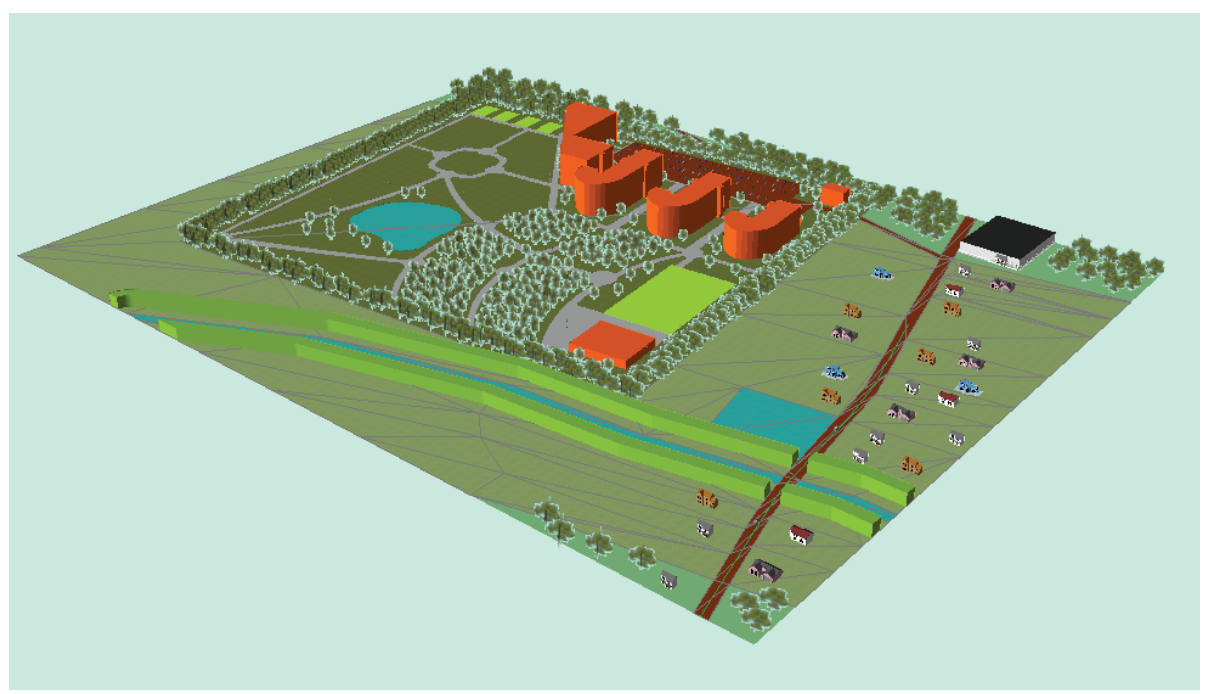

Fig. 2. Visualize the location and placement buildings designed holiday resort made in the software ArcScene 10

\section{Visualization Capabilities Using the Algorithm for Rendering in CAD Programs}

CAD programs are designed for presenting objects in a 3D environment. For many years, have developed a method for improving image quality by rendering three-dimensional graphic. This is the process of creating a two-dimensional image based on 3D model data. It consists in a series of simulations of physical effects using mathematical algorithms that describe them and see the results of calculations in the form of suitable image. In the algorithm exist simulations of natural and artificial lighting, light reflections, transparency, refraction, and a number of effects improving rendered scene [2].

In short, this is a photo 3D scene containing three-dimensional data. In the past, rendered objects were simple, built from basic shapes cube, sphere, planes, cylinders, and cones. This was due to the low computational power of computers. Over time began the dynamic development of 3D graphics caused by a technological progress. Nowadays, we are able to present a 3D scene consisting of many of objects, which may be buildings, trees, cars.

It is possible to generate and visualize the entire ecosystem of vegetation, inter alia, forests, cities with population and traffic, the sea, lakes and many other. To show the level of advancement of the algorithm Advance renderer used in the program Cinema4D, will be presented his short characteristics. Please note that the topics discussed below also apply to all the available algorithm renderers and operation in various $\mathrm{CAD}$ applications is same. 
The first and most important physical effect is lighting scenes using natural light of the sun and sky. Lighting the sun is implemented using the spotlight, significant distance away from the plane of incidence, so that the rays are parallel to each other, shadows of objects are arranged like in nature in the same direction. This light in the program Cinema4D has been named as the Sun. It is also available in more elaborate option Physical Sky. Illuminating the scene we have a variety of options, inter alia, the quality and type of shadows, lighting power, position of the sun regulated by the calendar (Sun can be set to the correct position for any geographic coordinates) and the color and saturation. The shadows are generated using ray tracing algorithm. This involves sending a single beam of light and an analysis of its course. If it hits an obstacle opaque terminates its run, followed by a dark fragment formed called shadow. Lighting the sky is a good way to simulate weather without sunlight, cloudy. Shadows at the light are ignored, and the light emitted by whole sphere model of the sky. The light is scattered and unfortunately not highlights the 3D depth effect. Artificial lighting is based on emission of three types of lighting: spot, directional and surface. His task is to present the different sources of artificial light, including light bulbs, reflector and fluorescent lamps. The light spot, due to that is close to the surrounding objects, shadows around emits, they are not parallel as in the case of sunlight. Light spot shines in one direction. Creates a cone beam of light with a opening depending on the user. Only items staying in the cone of the light will be lighted and generate shadows. The last type of light is the light surface, which due to the large surface emitting rays creates very soft shadows. Well suited to imitate the room illumination using fluorescent lamps. Generating light by sending rays of lighting points and the surface does not create fully photorealistic lighting. The most important effect that gives the reality of the scene is a global illumination effect that simulates any reflection, scattering and refraction of light.

In the Advanced Renderer, we can choose two ways of calculating global illumination: Irridiance Cache and Quasi Monte Carlo. The first model creates records on visible surfaces in a given range. Each such record will be sent in any direction Stochastic Samples (random rays). Each ray collects information about the color and strength of the lighting, which read, falling to the surface and creates an average power of color and lighting of the record. The second method, called shortly QMC consists in creating one big record for the entire scene seen by the camera. This requires change the settings a large number of samples to delete the grain, so rendering using this algorithm significantly extends [5].

Another useful effect is the Ambient Occlusion. It consists in obfuscation suitable places, creating shadows without light sources. The basis of the algorithm is a virtual ray that is sent from the plane to find other the nearest. If it will find cause shading that part of the plane from which it was sent. The degree of opacity and color is affected by many factors, including distance and the walls the angle of connection. This effect highlights the connection objects from the ground and various small parts [7].

The option that improves perception presented scene is undoubtedly the ability to smooth the edges of the escalator through antialiasing algorithm. It is still being developed. Allows smooth the edges of objects. Today it is possible to smoothing 
transparent elements and textures on surfaces. Without it, the visualization of certain types of analyzes cannot be properly understood.

For enhancing the attractiveness of our scenes, we can use a number of simulations of atmospheric physics effects, which are polluted air, fog, smoke, water vapor and clouds. These effects allow us to get better control over the photorealism scene, emphasizing the problem and simulation of phenomena occurring on the visualized area. Examples are air pollution in the city, moving fog in mountain ranges, fire and smoke in areas affected by the fires.

Using multiple effects it also has its drawbacks. For one of them we can include rendering time. Some scenes require generation of a few hours. Another downside is that the excess of different effects can obscure the main issue presented in the image of the analysis performed. Therefore, all effects and glamourizing the scene should be chosen carefully in order not to unnecessarily lengthen the rendering time and not to overshadow, but highlight the problem presented. In Figure 3 we can see an example of the algorithm renderer, contained in the CAD software, in the process of visualization objects holiday village. The picture was created using the GIS spatial data about the location of each element, and objects (trees, texture) were added at a later stage in the CAD software Cinema 4D. Time image rendering lasted about 30 minutes.

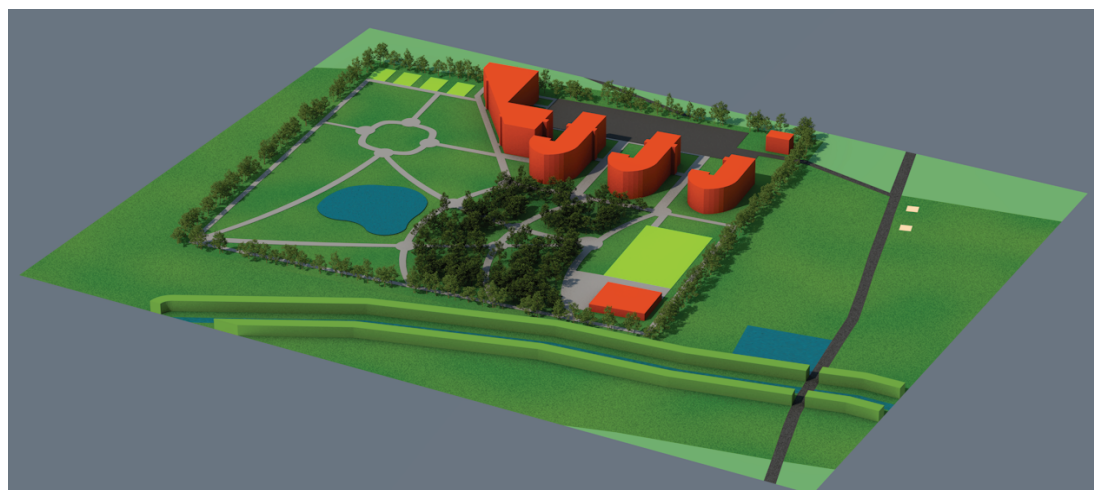

Fig. 3. Visualize the location and placement buildings designed holiday resort

\section{Cooperation ArcGIS Software with the Program Cinema4D}

By doing the analysis of various types, to a lot of software, we are not forced to use for everything only one software. There is no question that the program will ArcScene better suited to do analyzes, prepare data for visualization, while the rendering algorithm application Cinema4D - to generate an image presenting the results of analyzes. 
When creating 3D scene in ArcScene application, we can one step to export it as a file VRLM (*.wrl) and then load into the program Cinema4D. The only problem in the import file is no point elements and symbols, which may in certain projects complicate the process of importing data. Easily export a layer of 3D geometry and polygons and with information about the colors.

The scene before rendering can remodel, adjust color and lighting, insert additional objects or program a sequence of images if we want to create an animation. The finished rendering can be saved as an image or movie file in any size of up $128000 \times$ 128000 pixels. In Figures 4-6 show examples of the visualization software ArcScene prepared, and using an algorithm included in the software renderer Cinema4D. In part " $a$ " of the drawings used GIS software family ArcScene, and part " $b$ " was used to build an algorithm for rendering an image from an application Cinema4D. Image rendering time (part " $\mathrm{b}$ ") was for Figure 41.5 minutes, to Figure 5 of about 20 minutes, and for Figure 6, the rendering time exceeded 40 minutes.
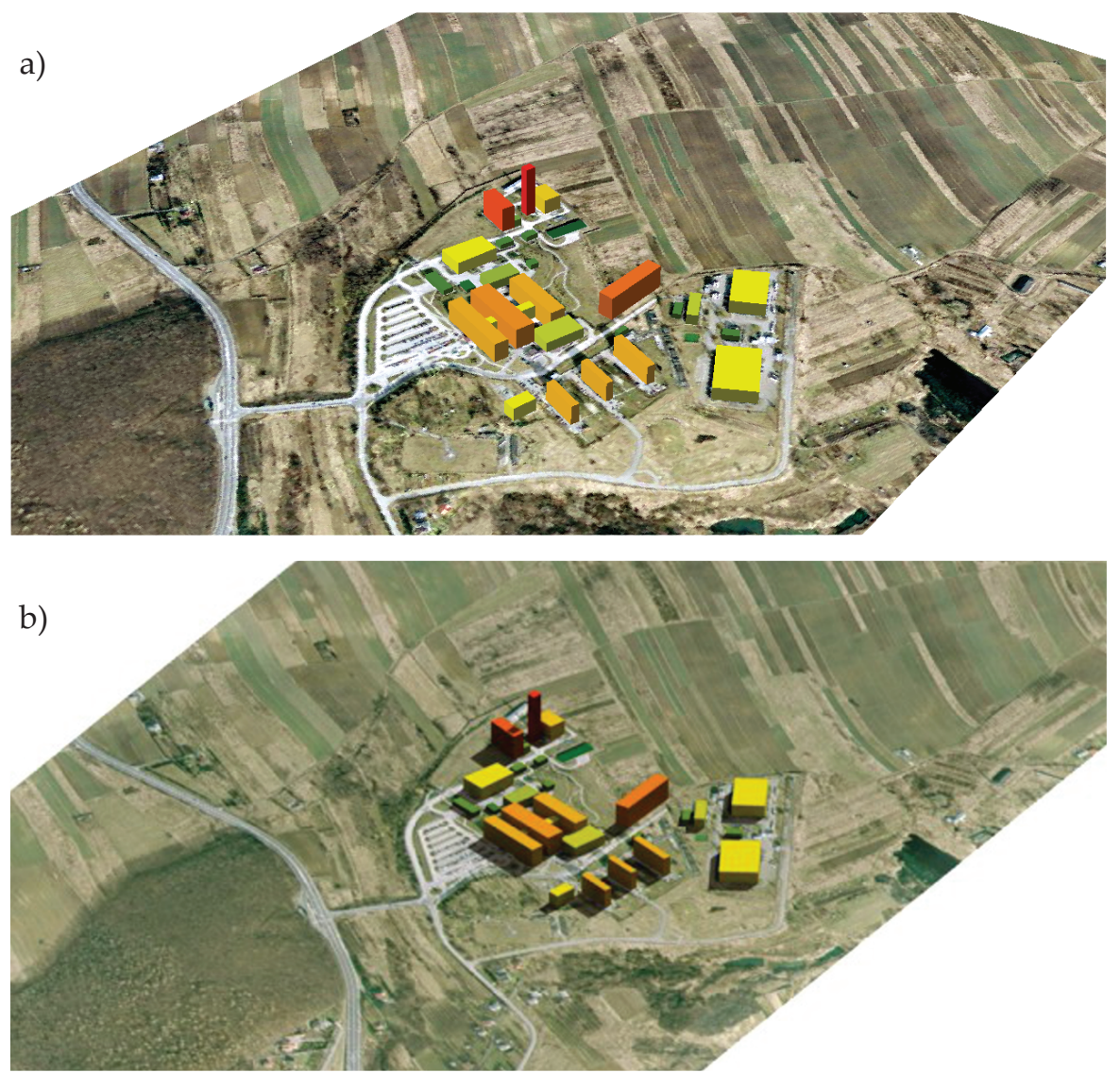

Fig. 4. Visualization building with accent on different heights of buildings using the color palette 
a)

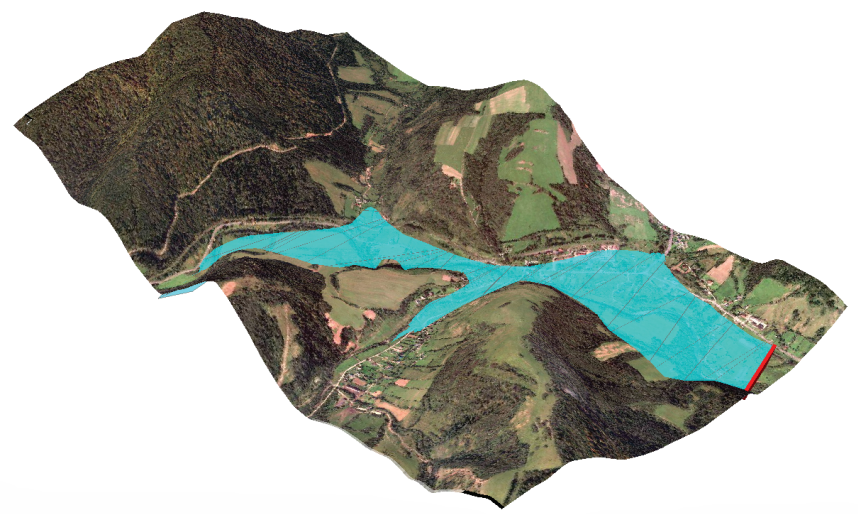

b)

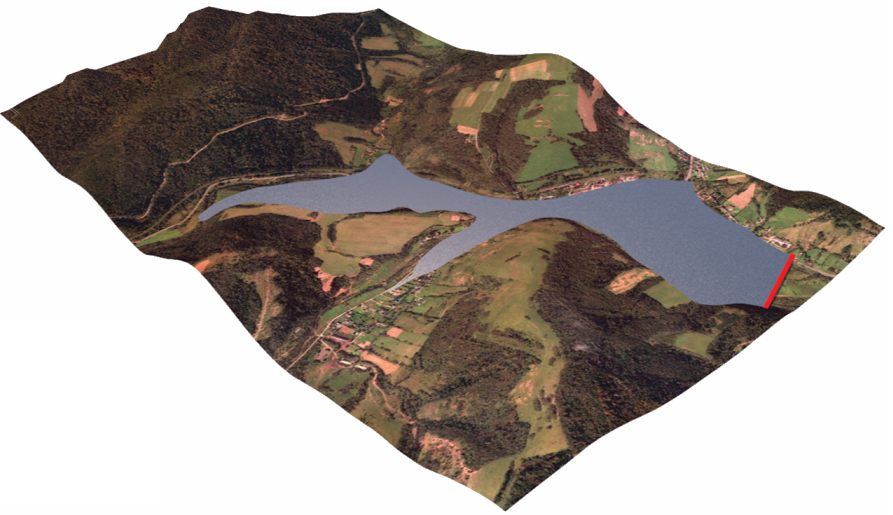

Fig. 5. Visualization of water level and landscape of a lake. In both cases, the surface of the field was used to generate digital terrain model in the form GRID

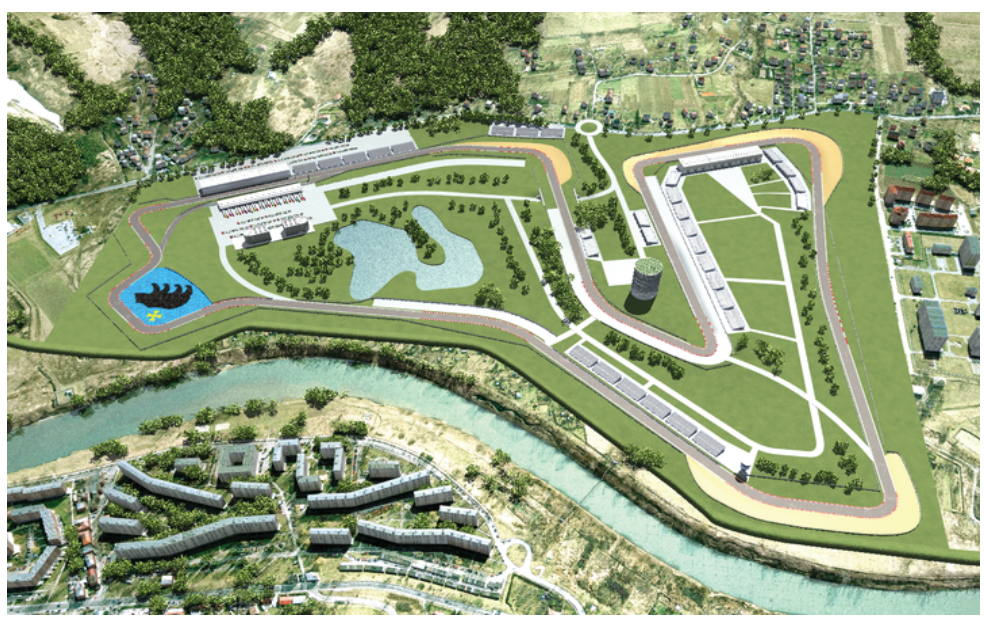

Fig. 6. Visualization of the city Przemyśl with applying the investment racetrack 
In Figure 5 we can see the advantage of CAD applications, namely water reflections that may have more attractive image. In Figure 6, we can see that during implementation of advanced visualization are used, inter alia, 3D objects as buildings, trees, and other objects of land use. All figures have been prepared by the author.

\section{Conclusions}

After reviewing scenes generated directly from ArcGIS, and the same scenes undergoing the process of rendering in CAD applications, we can conclude that the presentation of 3D data is preferable to use the duo programs. This allows us to perform the image more comprehensible to the recipient. The picture is easier to read visualization becomes more vivid. When creating animation in CAD applications we have more control over the movement of individual objects, where GIS applications are only available its base. A drawback of this type of connection may be time rendering of scenes, but the author believes it is worth to spend even a few hours on rendering to the image presented was definitely better.

Analyzing the two types of software, we can see some similarities. The same generating shadows can be considered a function of GIS (visibility). Undoubtedly, new CAD programs are emerging advanced simulations of water flow (RealFlow). They will allow not only the real visualization of flowing water in rivers, but also show us which way a given mass of water will move around the terrain model, for example, during a flood. CAD software will be able to partially fulfill the functions of GIS program.

Despite many similarities in GIS and CAD programs still need to integrate the two environments to achieve the advanced effects work. Using GIS program creates a base for appropriate presentation of data and to achieve the best possible effects, GIS software should support the CAD tools, especially in tasks where GIS has less utility as shown in the examples 3D visualization of analysis results.

The big downside of CAD software is a long time to render and export the scene. The application ArcScene allows the exporting the scene, but some GIS applications do not have this option. Additionally, if there is a change in the whole process of 3D scene visualization objects we need to do from the beginning. Therefore, a new branch of software, used visualization of 3D objects using graphics engines operating in real time. To their families we can include TerrainView [1] and Google Earth. These are programs which derive needed resource of 3D data from a database and perform their visualization (most frequently through OpenGL algorithm). The advantage of such applications is time rendering, good integration with GIS systems and the ability to visualize the presentation on the Web. The greatest future have applications allow flexible real-time rendering, and allowing use with databases containing 3D objects. In this case, GIS systems are supported by an external rendering algorithm, allowing up to date visualize changing data during the analysis, generated by any software for spatial analysis. 


\section{References}

[1] Beck M.: Real-Time Visualization of big 3D City Models. The International Archivesof the Photogrammetry, Remote Sensing and Spatial Information Sciences, vol. XXXIV-5, 2003, pp. 1-6.

[2] Birn J.: Cyfrowe oświetlenie i rendering. Wydawnictwo Helion, Gliwice 2007.

[3] GIS Support - Systemy Informacji Przestrzennej, [on-line:] www.gis-support.pl/ [access: December 2012].

[4] Litwin L., Myrda G.: Systemy informacji geograficznej: zarządzanie danymi przestrzennymi w GIS, SIP, SIT, LIS. Wydawnictwo Helion, Gliwice 2005.

[5] Orlikowski R.: Czym jest GI (globalna iluminacja)? [on-line:] www.c4d-pl.org/ modules.php?name=News\&file=article\&sid=791 [access: January 2013].

[6] TF3DM - 3D Models for free, [on-line:] www.thefree3dmodels.com [access: December 2012].

[7] Zapolski K.: Ambient Occlusion w Cinema 4D. [on-line:] www.zapolski.pl/ forever_after/?p=175 [access: January 2013]. 